\title{
Patient counseling with computer assisted instruction versus traditional diagrams for stress urinary incontinence and pelvic organ prolapse
}

\begin{abstract}
Background: Computer assisted instruction (CAI) for patient counseling has emerged as an alternative to traditional instructional diagrams (TD). As most pelvic floor disorders are treated electively, CAI provides an option for educating patients with urogynecologic conditions.

Methods: 56 patients diagnosed with SUI and/or POP were recruited from an academic urogynecology practice between December 2016 and June 2017. Patients were counseled on their diagnosis and treatment options using TD, and subsequently by a web-based CAI. Patients completed a Likert-style survey with questions to assess preference and satisfaction with each counseling modality.

Results: Regarding patient satisfaction with comprehension of diagnosis and treatment options, there was a significant difference in the proportion of patients who were satisfied with CAI and unsatisfied with TD $(48.9 \%)$ vs. those satisfied with TD and unsatisfied with CAI (2.2\%). Overall, significantly more patients preferred counseling with CAI vs. TD ( $93 \%$ vs. $4 \%$ ), and CAI had significantly greater impact on patients' medical decision making ( $91 \%$ vs. $5 \%)$.

Conclusion: Patients expressed higher levels of satisfaction with counseling using CAI versus TD for their condition and management of SUI and POP. Employing CAI for patient counseling for treatment of SUI and/or POP, may facilitate the patient decision making process for management.
\end{abstract}

Keywords: computer assisted instruction, counseling, pelvic organ prolapse, stress urinary incontinence, traditional diagrams
Volume 10 Issue I - 2019

\author{
Nicole M Golbari,' George Lazarou,2 Bogdan \\ Grigorescu² \\ 'Stony Brook University School of Medicine, Stony Brook, USA \\ ${ }^{2}$ NYUWinthrop University Hospitals, Mineola, USA
}

\begin{abstract}
Correspondence: Bogdan Grigorescu, MD, NYUWinthrop Women's Contemporary Care Associates, 120 Mineola Blvd \#I00, Mineola, NY I I50 I, USA, Tel + I-516-663-3010, Email BGrigorescu@nyuwinthrop.org
\end{abstract}

A portion of this data has accepted for podium Oral Presentation at the American Urogynecologic Society Pelvic Floor Disorders Week, Providence, RI. October 3-7, 2017

Received: November 12, 2018 | Published: January 17, 2019

\section{Introduction}

Pelvic floor disorders comprise a group of clinical conditions that impose a significant burden of disease. ${ }^{1}$ An estimated 160 million American women currently suffer with urinary incontinence, and more than $50 \%$ of these women have symptoms of stress urinary incontinence (SUI), in particular. ${ }^{2}$ In addition, up to $41 \%$ of women with, and $38 \%$ of women without a uterus have some degree of pelvic organ prolapse (POP). ${ }^{3}$ About half of all symptomatic women seek medical care for SUI and/or POP, and 1 in 5 women will have corrective surgery by the age of $80 ., 5$ As most corrective surgery is elective, these estimates underscore the importance of adequate patient education and encouragement to actively participate in the decision making process for management of SUI and/or POP. However, some studies suggest that women may be under-informed and ineffectively counseled prior to pelvic surgery. ${ }^{6}$

Computer assisted instruction (CAI) has emerged as an alternative to oral explanations and standard instructional diagrams or drawings. In contrast to the typical office-based counseling, CAI can combine auditory, visual and interactive methods to reinforce an educational message. This can be an especially useful tool among patients with education barriers or low health literacy as it standardizes the material being presented and allows patients to proceed at their own pace.

Several studies have demonstrated that CAI can increase patients' knowledge about health topics and assist in patient satisfaction during the informed consent process for surgery. ${ }^{7,8}$ Recent randomized trials have even suggested that patients are able to retain more information from a computer based education system alone than from static illustrative diagram-based instruction provided by their physician. ${ }^{9}$ However, there is a paucity of published studies examining patient counseling using computer based education programs for urogynecologic conditions. ${ }^{10,11}$ As patients with disorders such as SUI and POP tend to be older, CAI may be helpful for the explanation and understanding of the complex corrective surgical procedures for these conditions, yet the utility of this counseling modality is not well delineated. The primary objective of this study was to compare patient preference for counseling method with regards to patient comprehension of their diagnosis. The secondary objective was to assess the counseling method that best helped patients reach a decision regarding treatment. We hypothesized that, when presented with both methods of counseling, patients would express preference for CAI and CAI would be more influential in assisting patients to make medical decisions.

\section{Methods}

The Institutional Review Board at NYU Winthrop-University Hospital approved this study. Patients included in this study were all female patients with SUI and/or POP severe enough to merit surgical intervention that were seen in the urogynecology division at NYU Winthrop-University Hospital between December 2016 and June 2017. All patients included in the study had adequate command of English language, without need for interpreter. We excluded patients 
with cognitive impairment, and patients who needed a foreign language translator.

Subjects' age, race, and diagnoses were recorded. All patients were counseled regarding diagnosis and treatment options, specifically surgical treatment, for SUI and/or POP using traditional diagrams (TD) from Boston Scientific with relevant pelvic floor anatomy images, followed by counseling with a web based CAI [http://bscipopq.com/intro.html (C)2017 Boston Scientific Corporation] guided by the treating physician on a desktop computer (Appendix 1). Both methods of counseling were provided by the treating physician and outside of the visual aids employed, the verbal counseling was the same for both methods. After receiving both counseling methods, patients gave verbal consent to the study, and completed an eightquestion survey (Figure 1). Questions 1-5, which asked patients to rate their satisfaction with the counseling methods provided, were adapted from the questions in the validated Satisfaction with Decision Scale. ${ }^{12}$ The last three questions were designed as a head to head comparison of counseling modalities in regards to ease of understanding, mode of information delivery, and assistance with health care decision making. The investigators added the eighth question, regarding the impact of counseling method on medical decision-making, after patient enrollment began.

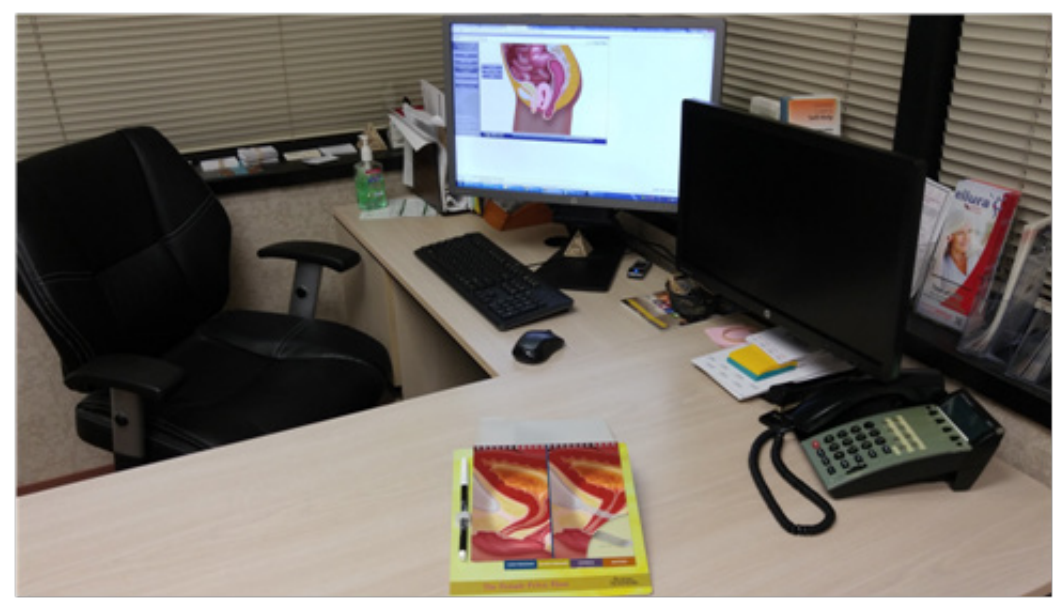

A)
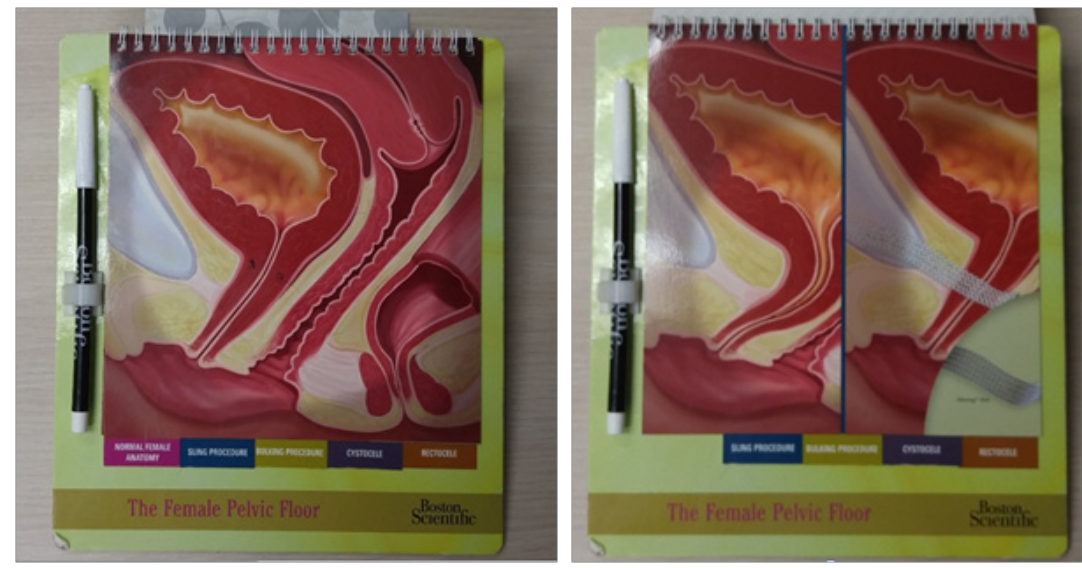

B)
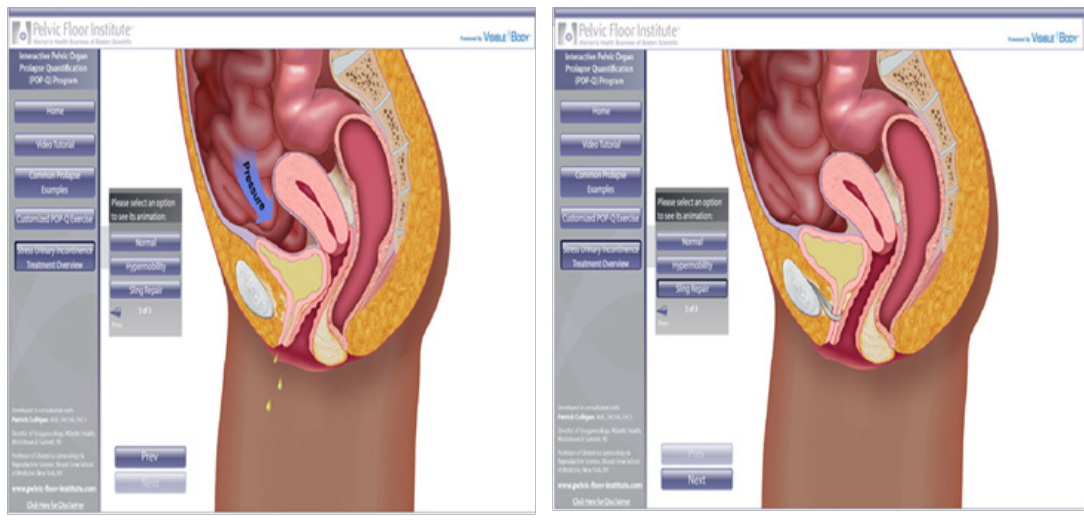

C)

Appendix I (color should be used in print if possible): A) Set up of counseling study. Examples of B) Traditional Diagrams (TD) and C) Computer Assisted Instruction (CAI) used for in office counseling of stress urinary incontinence (SUI). 


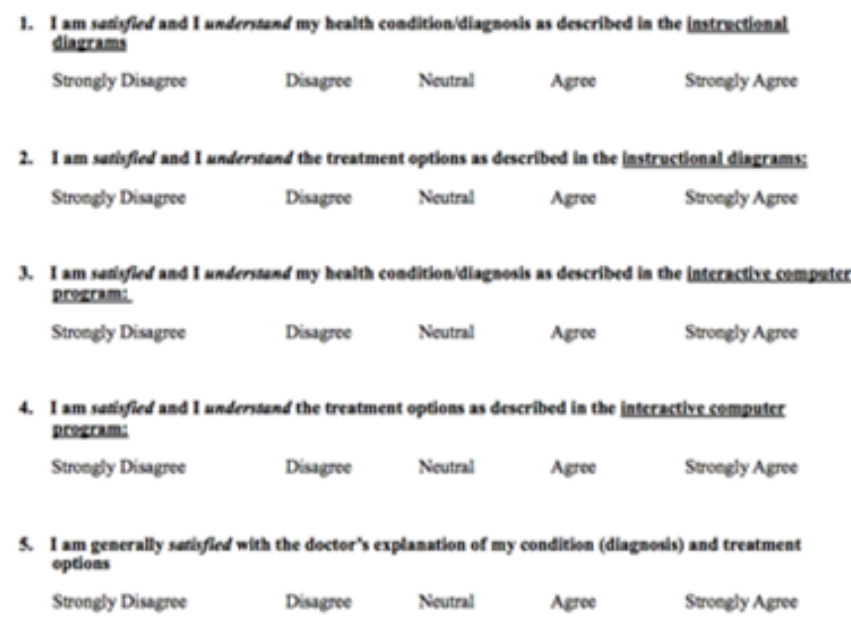

Figure I Patient satisfaction survey.

Responses from question 1-4 were dichotomized and the McNemar paired proportion test was used to compare the proportion of patients who are satisfied with the TD and not CAI, vs. the proportion of patients who are satisfied with the CAI and not the TD. We further stratified the Likert questionnaire results and patient preference for TD vs. CAI, based on patient age and the condition they were diagnosed with (SUI and/or POP). Analysis was done using SAS 9.4®. Two tailed chi-square and Fisher exact probability tests were used to compare patient preferences for TD vs. CAI in questions 6-8. P-values $\leq 0.05$ were considered significant.

\section{Results}

A total of 56 patients completed the survey with a mean age of $57.9 \pm 13.6$ years. Patient demographic data was available for 52 subjects and are presented in Table 1.

Regarding patient diagnoses, $42 \%$ of patients had SUI, $41 \%$ had POP and $8 \%$ had both conditions. Responses to the Likert scale questions indicated higher frequency of patients were strongly satisfied with their understanding of their health condition (93\% vs. $23 \%$ ) and treatment options ( $93 \%$ vs. $20 \%$ ) when counseled with CAI as opposed to TD (Figure 2). Comparing question 1 to question 3 , there was a significant difference in the proportion of patients who were satisfied with TD and unsatisfied with the CAI (proportion $=1.8 \%$ ) vs. the proportion of patients who were satisfied with the CAI and unsatisfied with the TD

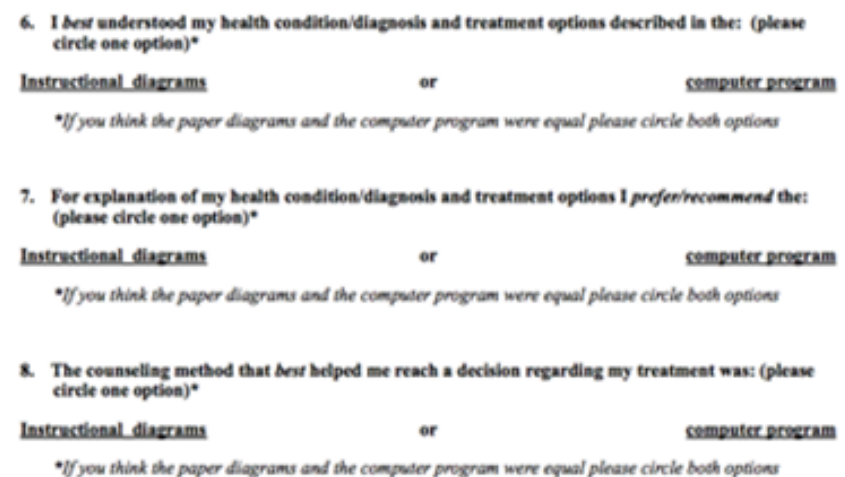

(proportion $=46.4 \%$ ), $\mathrm{p}$-value $<0.001$ as a method of understanding the diagnosis. Comparing question 2 to question 4 , there was a significant difference in the proportion of patients who are satisfied with TD and unsatisfied with the CAI (proportion $=0.0 \%$ ) vs. the proportion of patients who are satisfied with the CAI and unsatisfied with the TD (proportion $=46.4 \%$ ), $p$-value $<0.001$ as a method of understanding the treatment.

Table I Patient Demographics

\begin{tabular}{ll}
\hline $\begin{array}{l}\text { Age mean (SD) } \\
\text { Race n (\%) }\end{array}$ & $57.9(13.6)$ \\
Caucasian & $32(61 \%)$ \\
Black & $8(15 \%)$ \\
Asian & $2(4 \%)$ \\
Hispanic & $6(12 \%)$ \\
Unknown & $4(8 \%)$ \\
Diagnosis n (\%) & \\
SUI & $22(42 \%)$ \\
POP & $21(41 \%)$ \\
Both SUI and POP & $9(17 \%)$ \\
\hline
\end{tabular}
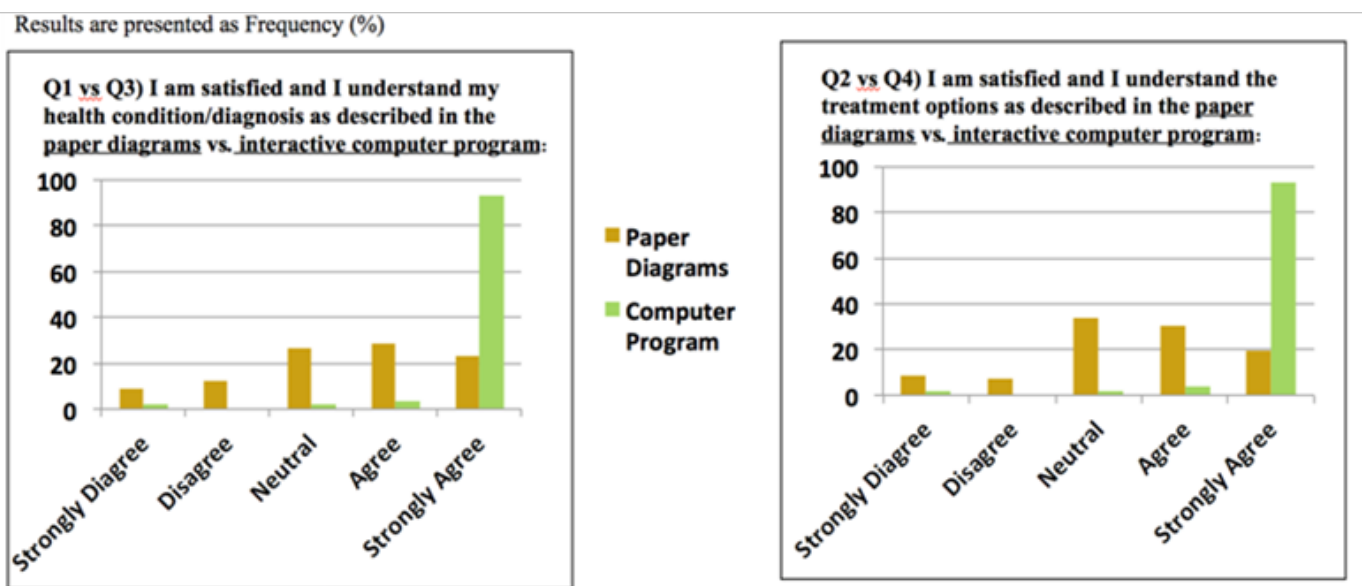

Figure 2 Understanding of diagnosis (QI vs Q3) and Treatment options (Q2 vs Q4). 
Additionally, more patients preferred CAI to TD for the explanation of their diagnosis and management options $(\mathrm{p}<0.0001)$ (Figure $3 \mathrm{a})$ and expressed greater understanding of their condition after counseling with CAI $(p<0.0001)$ (Figure $3 b)$. In order to better understand which counseling method had greater impact on treatment decisionmaking, we added an eighth question to our survey. Importantly, of the 48 patients that responded to the added question, $88 \%$ expressed that CAI was the method of counseling that most helped to guide treatment decisions (compared to $(\mathrm{p}<0.0001)$ (Figure $3 \mathrm{c}$ ). In order to best elucidate results, patient responses were analyzed by condition (SUI and/or POP), and patient's age.

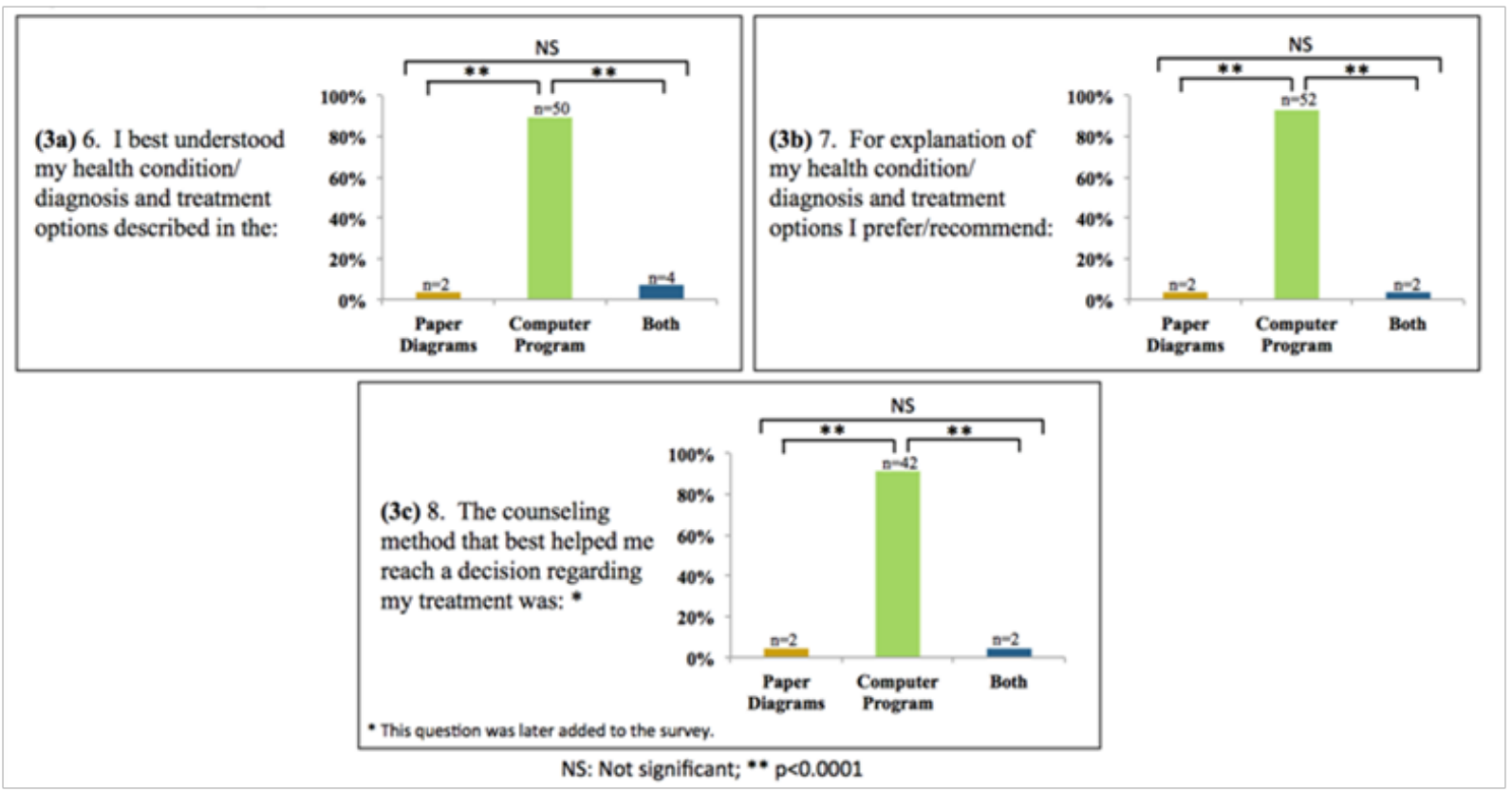

Figure 3 Patient preferences TD vs CAl.

Patients who were 60 years of age or older $(n=24)$, and patients who were younger than 60 years of age $(n=28)$ showed similar preference for CAI versus TD when asked which counseling method helped them understand their health condition/diagnosis and treatment options $(\mathrm{p}=0.28)$. Similar results were observed for answers to questions 7 and 8 of the questionnaire, $\mathrm{p}=0.45$ and $\mathrm{p}=1.0$, respectively. Additionally, most patients showed preference for CAI vs. TD when results were stratified by patient condition (SUI vs. POP).

\section{Discussion}

To our knowledge, this is one of the first few studies to report on patient preference for counseling method on SUI and POP conditions, and the impact of counseling modality on patient decisionmaking process for surgical treatment. Of the two previous studies published on the use of CAI for urogynecologic conditions, Bulmer et al. ${ }^{10}$ determined that CAI was able to increase patient knowledge of symptoms related to overactive bladder. ${ }^{10}$ The other study, a randomized control trial by Myers et al., ${ }^{11}$ examined the impact of CAI on satisfaction with counseling and patient anxiety related to POP symptoms. They determined that CAI was able to improve patient satisfaction and decrease anxiety related to their POP diagnosis as compared to traditional counseling with pamphlets. As opposed to the study from Myers et al. ${ }^{11}$ in this study, we included patients with both POP and SUI diagnoses. The patients were exposed concomitantly to both counseling methods, allowing for head-to-head comparison of TD and CAI. Our study is consistent with the previous reports of patient preference for CAI counseling. However, we also demonstrate that CAI can have a significant impact on not only the understanding of diagnoses, but also ultimately on the treatment decision-making process for patients with POP and SUI.

\section{Conclusion}

Results from this study suggest that CAI and other digital based medical education tools can have a significant impact on patient satisfaction with counseling and patient health care decision making. Though older patients tend to not be as familiar or comfortable with computer based educational tools, particularly in a health care related setting,,$^{13}$ we found that, even when stratifying for age, patients expressed a substantial preference for CAI counseling methods for understanding diagnosis and surgical treatment options for the management of SUI and POP. This study may indicate that CAI can be used to increase patient comprehension of their SUI and/ or POP diagnosis. Moreover, as patients expressed that CAI was more influential in helping patients decide on treatment preference, employing CAI for patient counseling prior to surgical intervention for SUI and/or POP, may help facilitate the patient's decision-making process.

Study limitations include small number or patients enrolled due to time constraints during office hours. Moreover, due to geographic constraints, our study population was predominantly Caucasian, and therefore results may not be applicable to other demographic areas. Additionally, due to the desire for head-to-head direct comparison of the counseling methods, patients were not randomized and investigators were not blinded, which may introduce bias. Nevertheless, as results of this study are persuasive, further confirmatory investigations are warranted. Future directions should include testing patient understanding of their diagnoses with a basic quiz before and after counseling with CAI vs. TD.

While discussion and consultation with the surgeon will always be essential in a patient's decision making, CAI may increase patient 
comprehension regarding not only the diagnosis of their condition, but also of the surgical treatment options available. ${ }^{14}$ Moreover, employing CAI for patient counseling may help facilitate patient ability to participate in the decision making process for management of their conditions. Replacing traditional paper diagrams with CAI for in office patient counseling, may help improve patient comprehension of SUI and POP diagnosis and treatment options, thus may improve/ reinforce the informed consent process prior to surgical management of these conditions. In addition to the benefits for patients, including enhanced autonomy and assurance in medical decision-making, using CAIs provides specific and customized treatment plan and may have a beneficial impact on patient satisfaction after surgical treatment, as previous studies have demonstrated that patients who receive comprehensive counseling are less likely to file malpractice claims. ${ }^{15}$ Future directions may include examining patient post-operative satisfaction after counseling with CAI vs. TD to further explore this impact.

\section{Funding details}

The authors have no commercial relationship to Boston Scientific and no competing interests to declare.

\section{Acknowledgments}

The authors would like to thank Dr. Rose Calixte for her contributions and Dr. Vintzileos for his support.

\section{Conflicts of interest}

Authors declare that there is no conflicts of interest.

\section{References}

1. Wu JM, Vaughan CP, Goode PS, et al. Prevalence and Trends of Symptomatic Pelvic Floor Disorders in U.S. Women. Obstet Gynecol. 2014;123(1):141-148.

2. Markland AD, Richter HE, Fwu C-W, et al. Prevalence and Trends of Urinary Incontinence in Adults in the United States, 2001 to 2008. $J$ Urol. 2011;186(2):589-593.

3. Hendrix SL, Clark A, Nygaard I, et al. Pelvic organ prolapse in the Women's Health Initiative: gravity and gravidity. Am J Obstet Gynecol. 2002;186(6):1160-1166.
4. Harris SS, Link CL, Tennstedt SL, et al. Care seeking and treatment of urinary incontinence in a diverse population. $J$ Urol. 2007;177(2):680684.

5. Wu JM, Matthews CA, Conover MM, et al. Lifetime Risk of Stress Incontinence or Pelvic Organ Prolapse Surgery. Obstet Gynecol. 2014;123(6):1201-1206.

6. Pakbaz M, Rolfsman E, Lofgren M. Are women adequately informed before gynaecological surgery? BMC Womens Health. 2017;17(1):68.

7. Cornoiu A, Beischer AD, Donnan L, et al. Multimedia patient education to assist the informed consent process for knee arthroscopy. ANZ J Surg. 2011;81(3):176-180.

8. Batuyong $\mathrm{E}$, Birks $\mathrm{C}$, Beischer AD. The use of multimedia as an adjunct to the informed consent process for ankle ligament reconstruction surgery. Foot Ankle Spec. 2012;5(3):150-159.

9. Keulers BJ, Welters CF, Spauwen PH, et al. Can face-to-face patient education be replaced by computer-based patient education? A randomised trial. Patient Educ Couns. 2007;67(1-2):176-182.

10. Bulmer PJ, James M, Ellis-Jones J, et al. A randomized trial comparing the effectiveness and preference of a touch-screen computer system with a leaflet for providing women with information on urinary symptoms suggestive of detrusor instability. BJU Int. 2001;88(6):532-535.

11. Myers EM, Robinson BL, Geller EJ, et al. Randomized trial of a webbased tool for prolapse: impact on patient understanding and provider counseling. Int Urogyn Jour. 2014;25(8):1127-1132.

12. Holmes-Rovner M, Kroll J, Schmitt N, et al. Patient Satisfaction with Health Care Decisions: The Satisfaction with Decision Scale. Med Decis Making. 1996;16(1):58-64.

13. Levine DM, Lipsitz SR, Linder JA. Trends in Seniors' Use of Digital Health Technology in the United States, 2011-2014. JAMA. 2016;316(5):538-540.

14. Anderson EE, Newman SB, Matthews AK. Improving informed consent: Stakeholder views. AJOB Empir Bioeth. 2017;8(3):178-188.

15. Boyll P, Kang P, Mahabir R, et al. Variables That Impact Medical Malpractice Claims Involving Plastic Surgeons in the United States. Aesthet Surg J. 2018;38(7):785-792. 